\title{
Ins Kumpelhafte abgleiten oder ins Oberlehrerhafte kippen. Zu Strukturen der Form ins -hafte + V
}

Die Produktivität des Nominalisierungsprozesses im Deutschen eröffnet vielfache expressive Möglichkeiten. Sie ermöglicht z.B. in Kombination mit Präpositionalphrasen neue aspektuelle Ausdrucksformen (siehe etwa Progressivformen wie am Überlegen sein oder Funktionsverbgefüge zum Kochen bringen). Im vorliegenden Beitrag werden nominalisierte Formen von haft-Adjektiven in Strukturen der Form: ins ${ }^{\star}$ hafte + Verb ${ }^{1}$ untersucht, wie sie in folgenden Beispielen aus dem Deutschen Referenzkorpus (DEREKo) ${ }^{2} \mathrm{zu}$ finden sind:

(1) Nie wird einer der zahlreichen orchestralen Effekte überbelastet, nie kippt der analytische Blick wie etwa bei Simon Rattles Beethoven ins Oberlehrerhafte. (profil)

(2) Es gibt starke Szenen, etwa eine Scheck-Übergabe, wo Werner Galas und Klaus Lembke als Unternehmer wunderbar ins Loriothafte verrutschen. (Braunschweiger Zeitung)

(3) Der freundschaftliche Kontakt zum heutigen Bundeskanzler blieb bis heute erhalten. Er ist einer, der Freundschaften pflegt, ohne aber ins Kumpelhafte abzugleiten. (Falter)

Im Gegenwartsdeutschen bilden solche Strukturen ein, wenn nicht sehr häufiges, doch zumindest in bestimmten Textsorten wiederkehrendes Muster, das aufgrund seiner Expressivität, ${ }^{3}$ aber auch einer Tendenz zum stereotypen Gebrauch

1 Auch möglich sind Fügungen mit deverbalem Substantiv: „Ausflüge ins Opernhafte“ (St. Galler Tagblatt).

2 https://cosmas2.ids-mannheim.de/cosmas2-web (Stand: 23.11.2017). DeReKo = Deutsches Referenzkorpus.

3 Der Begriff Expressivität ist in der Linguistik nicht scharf definiert (siehe Legallois/François 2009; Gautier/Monneret 2010; Chauvin/Kauffer 2013). In diesem Artikel sind die zwei folgenden Merkmale gemeint: Auffälligkeit und Ausdruck einer subjektiven Perspektive. Legallois/François (2012: 209) betonen, dass nicht der Inhalt einer Aussage an sich expressiv sei, sondern ihre Form. Expressivität wird nicht explizit ausgedrückt, sondern ist in den betreffenden sprachlichen Formen ‘wahrnehmbar' („L'expressivité se montre, elle ne se signifie pas“). Sie manifestiert 
ins Auge fällt. Interessant $\mathrm{zu}$ beobachten ist die besondere Konstellation aus der Integration eines nominalisierten haft-Adjektivs in eine direktionale in-Präpositionalphrase und der Kombination dieser Präpositionalphrase mit einem transformativen Verb, ${ }^{4}$ die im Folgenden beschrieben werden soll. Dabei soll auch die Musterhaftigkeit solcher Gebilde gegenüber ähnlichen Strukturen überprüft und deren Gebrauch erläutert werden.

\section{Eigenschaften der Struktur}

Die Analyse beruht auf 200 Beispielen aus dem DeREKo. Eine Suche der Form 'ins + *hafte' ergibt im DEREKo 2356 Treffer, von denen ein guter Teil allerdings nichtnominalisierte Formen des Adjektivs enthält (z.B. „der kurze Flug ins frühlinghafte Mailand“), also hier nicht interessiert. Präpositionalgruppen der Form bis ins ${ }^{*}$ hafte wurden auch ausgesondert, da sie meines Erachtens eine andere Struktur darstellen und oft loser an das Verb gebunden sind. ${ }^{5}$ Für die vorliegende Analyse wurden außerdem nur die ersten 200 relevanten Belege aus dem DEREKo berücksichtigt. Im Korpus entspricht dies den ca. 300 ersten Treffern der Suche im DEREKo.

sich im Zusammenhang mit dem Gebrauch dieser Formen in einem bestimmten Kontext und in einer 'bestimmten diskursiven Praxis' (ebd.: 211f.). Diese Merkmale treffen auf die hier untersuchte Struktur zu.

4 „Ein transformatives Verb beschreibt [...] die Überführung eines Zustandes in einen anderen Zustand.“ (Fabricius-Hansen 1975: 21 nach Wunderlich).

5 Soweit das Korpus diese Beobachtung zulässt, dienen die Präpositionalgruppen bis ins -hafte zum Ausdruck der Steigerung (siehe Beispiel a). Damit sind sie syntaktisch meistens fakultativ (a'), außer das Verb (z.B. reichen in Beispiel b) verweist auf das Erreichen eines Endpunkts, der genannt werden muss. Im Unterschied zu den im vorliegenden Artikel untersuchten Strukturen ins -hafte $+V$ (siehe unten 1.3) muss das Verb keine Veränderung ausdrücken, wie auch Beispiel (a) zeigt.

(a) Es ist wohl diese Stärke, für die vor allem Sophie viel später bis ins Heldenhafte verehrt wurde. (Mannheimer Morgen)

(a') Es ist wohl diese Stärke, für die vor allem Sophie viel später verehrt wurde.

(b) Ob er sich die bis ins Krankhafte reichende Zuneigung seiner Landsleute zu ihren Haustieren beguckt (,Tierische Liebe“), Menschen in der intimen Zwiesprache mit Gott filmt (,Jesus, du weißt“) oder sich den Abgründen im Leben eines Models („Models“) widmet: Wer in einen SeidlFilm geht, der setzt sich Situationen aus, die er sonst eher meidet. (Hannoversche Allgemeine). 


\subsection{Vorkommen}

Bis auf fünf Belege, die Romanen entnommen sind, handelt es sich um Pressebeispiele. In drei Viertel (153) der Fälle sind die Beispiele (wie 1-2 oben) der Textsorte 'Kunstrezension' zuzuordnen, d.h. sie treten in einem sprachlichen Kontext auf, in dem Musik- und Theateraufführungen, laufende Kunstausstellungen, literarische und musikalische Neuerscheinungen und aktuelle Filme besprochen und kritisch beurteilt werden. Dem deskriptiven und bewertenden Gebrauch im Kontext der Kunstrezension nah sind im Korpus vereinzelte Vorkommen in Presseartikeln, in denen Modetendenzen kommentiert werden (4 Beispiele), z.B.:

(4) Hohe, schwere, ins Briketthafte tendierende Galoschen, die zu meiner Zeit Plateauschuhe hießen. (Focus)

\section{2 haft-Adjektive}

Fast immer tritt das Suffix -haft gemäß den Beobachtungen von Fleischer/Barz/ Schröder (2012: 336) an eine substantivische Basis. Ausnahmen sind im untersuchten Korpus lachhaft und flatterhaft mit verbaler Basis, krankhaft und roséhaft mit adjektivischer Basis. Die Daten zeugen von der Produktivität des Suffixes -haft: Neben einigen rekurrenten lexikalisierten Adjektiven: floskelhaft, grauenhaft, klischeehaft, märchenhaft, opernhaft, rätselhaft, sagenhaft, scherzhaft, wahnhaft sind auch viele nicht usuelle Bildungen anzutreffen: briketthaft, comichaft, folklorehaft, geräuschhaft, schlagerhaft, stillebenhaft, thrillerhaft, rentnerhaft, revuehaft. ${ }^{6}$ Diese werden meistens komparativ, etwas seltener 'ornativ' (siehe Fleischer/Barz/Schröder, 314ff. und 336f.) interpretiert: Sie bezeichnen eine Ähnlichkeit mit dem Referenten der substantivischen Basis und können mit einem wie-Vergleich umschrieben werden: wie ein Brikett, wie ein Comic, wie ein Schlager ... oder das Suffix -haft erhält die Bedeutung 'mit etwas behaftet', z.B. folklorehaft (mit Folklore behaftet). Auch können haft-Adjektive in adjektivischen 'ad-hoc'-Komposita auftreten: allgemein-vogelhaft, balkanesk-volksliedshaft, zoologisch-variétéhaft, magisch-märchenhaft.

6 Letztere findet man nicht im Wörterbuch. Im Unterschied zu manchen lexikalisierten Adjektiven (wie sagenhaft, grauenhaft) werden sie rein kompositionell interpretiert; allerdings kann sich die Interpretation ohne Einbeziehung des Kontextes als schwierig erweisen (siehe revuehaft, rentnerhaft, geräuschhaft). 
Semantisch lassen sich unter anderem folgende typische Bildungsmuster bzw. semantische Typen unterscheiden:

1. In zahlreichen Beispielen bezeichnet die substantivische Basis ein 'Genre' der Malerei, des Theaters, der Musik ... (comedyhaft, kunstliedhaft, stilllebenhaft, possenhaft, schwankhaft, comichaft, revuehaft, jazzhaft, variétéhaft, parabelhaft, gleichnishaft, karikaturenhaft, volksmusikhaft, romanhaft, auch märchenhaft).

2. Ab und $\mathrm{zu}$ ist die substantivische Basis ein Eigenname, der auf eine prominente Person verweist, die für einen Stil steht (Loriothaft, Richard-Claydermanhaft). In ähnlicher semantischer Funktion kann sie auch ein Stilelement von einem Kunstwerk (floskelhaft, klischeehaft, symbolhaft) bezeichnen.

3. In einigen Beispielen verweist das Adjektiv auf eine übermäßige Größe (ins Riesenhafte wachsen, vergrößern ...; ins Fieberhafte steigen; ins Sagenhafte wachsen).

\subsection{Verben}

Die Verben, die in der betreffenden Struktur verwendet werden, drücken einen Veränderungsprozess oder eine Tendenz aus. Es überwiegen intransitive Vorgangsverben, die transitiven Verben erscheinen meistens in Partizipialgruppen (vgl. 5), in der Passivform (vgl. 6) oder pronominal (vgl. 7), so dass ein eventueller 'Verursacher" der beschriebenen Veränderung meistens nicht genannt wird. In nur ca. 10 Prozent der Beispiele stehen transitive Verben in der Aktivform (vgl. 8):

(5) Tief und rauchig tönt es durch den Raum. Am Bühnenhorizont ins Riesenhafte vergrössert, grobkörnig schwarzweiss: eine Marguerite im Schutt. (St. Galler Tagblatt)

(6) Der Kampf des Mannes mit der Frau genauso wie mit dem Stier wird hinterfragt, bestückt mit teilweise makabren Details, deren Drastik durch die Statik des Formalen reizvoll ins Stillebenhafte transformiert wird. (Tiroler Tageszeitung)

7 Ein „Agens“ (siehe Liste der semantischen Rollen bei von Polenz 1988: 167-174), das die Veränderung auslöst. 
(7) Schumanns Andante und Variationen sind von einer unheimlichen Abgründigkeit, die sich später in Rachmaninows „Barcarole“ ins Spukhafte wendet. (Hannoversche Allgemeine)

(8) Die Blätter seien „durchgängig von einem experimentellen, innovativen Geist geprägt“ und offenbarten die Fähigkeit des Pop-Art-Künstlers, die Wirklichkeit ins „Ikonenhafte zu transferieren“, erklärte das Museum. (Rhein-Zeitung)

Im untersuchten Korpus treten manche semantische Typen von Verben häufiger auf, und zwar:

- $\quad$ solche, die den Eindruck vermitteln, als würden die beschriebenen Prozesse von alleine verlaufen und sich der Kontrolle durch ein Agens entziehen, z.B. rutschen und Ableitungen: abrutschen, verrutschen kommen 8 Mal vor, driften und Ableitungen $13 \mathrm{Mal}$; abgleiten bzw. gleiten $15 \mathrm{Mal}$, (seltener auch: ausufern, abschweifen),

- $\quad$ solche, die einen Veränderungsprozess beschreiben, der plötzlich und unvermittelt eintritt, z.B. kippen (12 Okkurrenzen), geraten (2 Okkurrenzen), seltener abstürzen, rücken, umschlagen,

- solche, die einen Prozess der Steigerung, z.B. vergrößern 8 Okkurrenzen (seltener: steigern, übersteigern, überhöhen, überzeichnen, aufblähen) oder umgekehrt des Verschwindens (sich verlieren, sich auflösen) bezeichnen.

Häufig sind es Bewegungsverben, doch nicht der Aspekt des Ortswechsels steht hier im Vordergrund, sondern derjenige des Zustandswechsels.

Weitere Verben drücken eine Veränderung, Verwandlung oder einen Übergang aus (übergehen, übersetzen, changieren, wechseln, verklären, zerlegen, transformieren, übertragen, erweitern, wenden), nicht selten mit negativer Konnotation (entarten, verzerren, verziehen).

\subsection{Was leistet die Struktur?}

Die Struktur ins ${ }^{\star}$ hafte + V ermöglicht, der subjektiven Wahrnehmung eines laufenden Veränderungsprozesses oder einer Tendenz einen sprachlichen Ausdruck zu verleihen. Dabei wird der Prozess etwas vage und vorsichtig beschrieben und ist möglicherweise nicht ganz einfach in Worte zu fassen. Aus dem Zusammenspiel zwischen transformativer Bedeutung des Verbs, Semantik der haft-Adjektive, Nominalisierungsprozess und direktionaler Bedeutung der Präpositionalgruppe ergeben sich etwas genauer folgende semantische Merkmale: 
1. Ausdruck eines laufenden Veränderungsprozesses: Die Struktur beschreibt, wie ein Referent gerade eine neue Eigenschaft gewinnt. So lässt sich der in den Beispielen (1) bis (4) beschriebene Vorgang grob mit dem Verb werden umschreiben: nie wird der analytische Blick oberlehrerhaft / WG und Klaus Lemke werden Loriothaft. / ... ohne kumpelhaft zu werden / Die Galoschen werden briketthaft. Weitere Merkmale dieses Veränderungsprozesses ergeben sich aus den unter 1.3 aufgelisteten Merkmalen der verwendeten Verben, welche typischerweise Vorgänge bezeichnen, die für den Beobachter als unkontrollierte, urplötzliche oder sehr langsame Phänomene schwierig zu erfassen sind und einen verschwimmenden Eindruck hinterlassen.

2. Gerichtetheit des Vorgangs und neue Kategorisierung: Die direktionale Präpositionalphrase verweist auf einen (hier nicht erreichten) Endzustand (wobei die Vorstellung eines gerichteten Vorgangs nicht unbedingt mit der Semantik des Verbs selbst transportiert wird, siehe z.B. Verben wie sich verlieren, übersteigern, vergrößern ..., die Prozesse beschreiben, welche nicht auf einen Endpunkt hin ausgerichtet sind). Sie beschreibt hier kein konkretes räumliches Ziel, sondern verweist auf eine Eigenschaft, die durch die Nominalisierung des Adjektivs zu einer Kategorie erhoben wird. Da sich diese Eigenschaft nur durch Vergleich und approximative Annäherung bestimmen lässt (Semantik des haft-Suffixes), bleibt diese Kategorisierung relativ vage. Es handelt sich eher um einen Versuch, eine neue Kategorisierung für eine Erscheinung vorzunehmen.

Die erwähnten Merkmale erinnern zum Teil an von Thim-Mabrey (2001, 2007) genannte, für Musikkritik typische Stilmittel, die „Expressivität signalisieren“: bildhafte Vergleiche, „Substantivierungen von Adjektiven, die bewirken, dass eine Eigenschaft oder ein Merkmal des Beschreibungsgegenstands als eine den Einzelgegenstand übergreifende, umfassende Dimension erscheint“ (ThimMabrey 2001: 163). Der häufige Rückgriff auf die Struktur 'ins *hafte + V' in Kunstrezensionen erklärt sich durch das kommunikative Ziel, der subjektiven Wahrnehmung eines Veränderungsprozesses eine sprachliche Form zu geben. 


\section{Zur Musterhaftigkeit und Spezifizität der Struktur}

\subsection{Stichwort 'Konstruktion'}

Handelt es sich bei der hier besprochenen Struktur um eine 'Konstruktion' im Sinne der Konstruktionsgrammatik? Auch wenn das primäre Ziel der vorliegenden Untersuchung nicht ist, eine konstruktionsgrammatische Analyse zu liefern, bieten wohl die allgemeinen Sichtweisen der Konstruktionsgrammatik (siehe z.B. Ziem/Lasch 2013) einen relevanten und ergiebigen Ansatz zur Beschreibung solcher sprachlicher Formen. Diese Formen teilen auch mit den 'klassischen' Forschungsgegenständen der Konstruktionsgrammatik wie sogenannten 'causedmotion-Konstruktionen's zwei typische Züge: häufige Umdeutung eines Bewegungsverbs (kippen, gleiten, rutschen) in eine aspektuelle Bedeutung (hier: Veränderung), Einfügen einer direktionalen Erweiterung als 'Ziel'. ${ }^{9}$

Deppermann/Elstermann (2008) und Dobrovol'skij (2011) diskutieren verschiedene Kriterien in Bezug auf die Anwendung und Ergiebigkeit des Konstruktionsbegriffs. Bei der hier untersuchten Struktur sind vor allem die Kriterien der Usualität d.h. der „,ausdrucksseitigen Prägung für die eine bestimmte Konzeptualisierung und kommunikative Funktion“ (Deppermann/Elstermann 2008: 130) und der Möglichkeit verschiedener Realisierungen eines syntaktischen Patterns durch die „Sättigung der lexikalisch nicht spezifizierten Slots“ (Dobrovol’skij 2011: 116) als definitorische Züge einer Konstruktion relevant. Das untersuchte Muster erfüllt beide Kriterien: Einerseits scheint es tatsächlich für die Sprecher als sprachliche Ressource zu fungieren, um in einem bestimmten Kontext kommunikative Ziele zu erreichen. Wie oben gesagt, wird dieses Muster wenn nicht ausschließlich, so doch präferiert in einer bestimmten Textsorte, nämlich der Kunstrezension verwendet und dient zur Beschreibung und sprachlichen Kategorisierung schwierig kategorisierbarer wahrgenommener Veränderungsprozesse. Andererseits eröffnet die Struktur im Rahmen eines festen syntaktischen Musters und trotz der wiederholten Verwendung mancher Verben und Adjektive einen

8 Siehe u.a. Goldberg (1995); Dalmas/Gautier (2013).

9 Eigentlich ist die Struktur ins -hafte + V den 'resultativen' Konstruktionen Goldbergs verwandt. Nach Goldberg (1995: 81-89) sind resultative Konstruktionen 'metaphorische Extensionen' der caused-motion-Konstruktionen. Für diese metaphorische Hypothese spricht auch die häufige metaphorische Verwendung von Bewegungsverben als Veränderungsverben im Englischen: The jello went from liquid to solid in a matter of minutes. / Bob fell asleep. / Bob went crazy. (Beispiele von Goldberg). 
relativ großen Spielraum für kreative Kombinationen. Die Struktur scheint eine strukturelle und pragmatische Verfestigung aufzuweisen, lässt aber eine kreative Kombinatorik zu.

\subsection{Zur 'Musterhaftigkeit' des Musters ...}

Eine Suche der Form 'ins *hafte + V' im DEREKo ermöglicht es, eine Reihe von Verben zu identifizieren, die in dieser Struktur häufiger auftreten (siehe oben 1.3.) und sie nach semantischen Gruppen zu klassifizieren. Sucht man wiederum gezielt nach Kollokationen mit diesen Verben, so stellt man fest, dass die Struktur 'ins *hafte + V' nur einen Sonderfall der Struktur 'ins + nominalisiertes Adjektiv + V' darstellt, etwa bei den Verben abgleiten oder kippen, die ich exemplarisch untersucht habe. Eine Suche im DEREKo ergibt viele Beispiele wie die folgenden:

(9) Ein Blick auf die Darsteller: Sie zeigten sich frisch-fröhlich, sympathisch und kindgerecht, ohne ins Kindisch-Anbiedernde abzugleiten. (Braunschweiger Zeitung)

(10) Alltägliche Situationen, die jäh ins Irreale kippen, kennzeichnen Stefan Banz' kurzgeschnittene Videoarbeiten, die ab morgen Samstag, 5. Dezember, im «Schichtwechsel» an der Landstrasse 73 in Vaduz zu sehen sind: Vorbegriffliches, ungeordnet Nichtbekanntes. (St. Galler Tagblatt)

Solche Beispiele weisen ähnliche semantische und pragmatische Merkmale auf wie die oben untersuchten (Ausdruck eines Veränderungsprozesses, Versuch einer sprachlichen Kategorisierung, sehr häufige Verwendung in Kunstrezensionen). Das Fehlen eines haft-Adjektivs scheint diese Beispiele nicht prinzipiell von dem unter 1. untersuchten Muster zu unterscheiden.

Im Korpus finden sich sogar manche Beispiele ohne nominalisiertes Adjektiv, die offensichtlich sehr nah an den hier analysierten sind:

(11) Unerbittlich verbreiten sie an Starparaden und Galaabenden jene Stimmung von Reichtum und Glamour, die nach der anschliessenden Tagesschau mit schöner Regelmässigkeit ins Gegenteil kippt. Warum interessiert die Prominenz? Und was macht das Tagblatt daraus? (St. Galler Tagblatt)

(12) Es ist auffallend, dass der FC Rorschach dieses Frühjahr in jedem Spiel unterschiedliche Leistungen erbringt. Entweder glänzt der FCR eine Halb- 
zeit lang, um dann ins Mittelmass abzugleiten, oder Höhen und Tiefen wechseln in kurzer Folge. (Neue Zürcher Zeitung)

Sollte man nicht anstatt der Struktur (bzw. 'Konstruktion') 'ins + nominalisiertes Adj. + V' eher eine viel unbestimmtere Struktur 'in + direktionale NG + V' postulieren? Dann müsste man aber diese Struktur irgendwie unterscheiden von etwa folgenden Beispielen, bei denen die syntaktische Oberflächenstruktur 'in + direktionale NG + kippen / abgleiten' eine ganz andere Interpretation erhält:

(13) Ein ebenso grosses Problem seien die riesigen Mengen an Chemiewaffen, die ins Meer gekippt worden seien.

(14) Mit der Scheidung habe es begonnen, dann sei er ins Rotlichtmilieu abgeglitten und habe Ilona getroffen.

Der Unterschied zwischen (11) und (12) einerseits und (13) und (14) andererseits ist offensichtlich: In (13) und (14) ist die Bedeutung der verbalen Struktur nicht (wie in 11-12 sowie den vorigen Beispielen) transformativ und kategorisierend, sondern konkret räumlich-direktional (eine Paraphrase mit werden ist nicht möglich). Um die Polysemie der Struktur 'in + direktionale NG + V' zu beschreiben, müsste man diese also um semantische Merkmale (etwa + abstraktes $\mathrm{N}$ vs. + konkretes $\mathrm{N})^{10}$ spezifizieren, was eine Untersuchung an verschiedenen Verben und an einem größeren Korpus erfordert.

\section{3 -haft-Adjektive und andere Adjektive}

Ob die Struktur bzw. 'Konstruktion' 'ins * hafte + V' ein eigenständiges Muster darstellt oder nur ein Sonderfall einer allgemeineren Struktur 'in + direktionale NG + V' ist, bedürfte also einer näheren Untersuchung. In den Korpusbeispielen kommt jedenfalls in die Position 'direktionale NG' in Verbindung etwa mit den Verben kippen und abgleiten sehr oft bzw. meistens ein nominalisiertes Adjektiv. Auch kann man davon ausgehen, dass es eine gewisse Tendenz von haft-Adjektiven gibt, in dieser Struktur aufzutreten. Dies erklärt sich meines Erachtens dadurch, dass nominalisierte Formen von haft-Adjektiven imstande sind, vage Kategorien

10 Offensichtlich ermöglicht das Verweisen auf einen abstrakten Referenten (sei es anhand eines 'abstrakten Nomens' oder eines nominalisierten Adjektivs) die metaphorische Umdeutung der gesamten Struktur (siehe Fußnote 9). 
herauszubilden und zu bezeichnen: Das 'Märchenhafte’ z.B. verweist nicht direkt auf das Genre des Märchens, sondern auf ein vages Bündel an Eigenschaften, die an Märchen erinnern/für Märchen charakteristisch sind ... Darüber hinaus macht es die Produktivität des haft-Suffixes möglich, neue und teilweise einmalige Kategorien zu schaffen, wie z.B. -haft-Adjektive, die mit einem Eigennamen gebildet werden: das Loriothafte, das Richard-Claydermanhafte. Aus beiden Gründen eignen sich haft-Adjektive besonders gut, um schwierig zu kategorisierende Phänomene wie Veränderungsprozesse $\mathrm{zu}$ beschreiben.

Andere Adjektivbildungen weisen ähnliche Eigenschaften auf, vor allem ad-hoc-Komposita, deren Interpretation sehr stark von dem Gebrauchskontext abhängt (siehe Beispiele 9, 15 und 16). Nominalisierungen von Adjektiven mit -esk und -mäßig-Suffixen haben in der besprochenen Struktur eine ähnliche semantische Leistung, allerdings werden sie viel seltener verwendet als -haft-Adjektive.

(9) Ein Blick auf die Darsteller: Sie zeigten sich frisch-fröhlich, sympathisch und kindgerecht, ohne ins Kindisch-Anbiedernde abzugleiten. (Braunschweiger Zeitung)

(15) Die Vitalität, der oft ins Trotzig-Rotzige kippende Schwung solcher Beschreibungen ist nicht alltäglich. (Braunschweiger Zeitung)

(16) Ohne sich solistisch zu profilieren oder ins Heldenhaft-Pathetische abzugleiten, spielte er einen Beethoven, der vor Lebensfreude und Zuversicht nur so strahlte. (St. Galler Tagblatt)

Eine Kookkurenzanalyse der Verben kippen und abgleiten anhand des in Cosmas vorhandenen Analysemoduls (siehe Belica 1995) zeigt allerdings, dass die häufigsten Adjektive keine haft-Adjektive sind. ${ }^{11}$ Mit kippen am häufigsten kombiniert werden Nominalisierungen folgender Adjektive: negativ, absurd, lächerlich, surreal, grotesk, komisch, kitschig, sentimental, hysterisch. Abgleiten wird zum Teil mit denselben Adjektiven kombiniert, die allerdings mit etwas anderer Häufigkeit verwendet werden: lächerlich (ins Lächerliche abgleiten ist die häufigste Kombination), sentimental, kitschig, absurd, banal, grotesk. Ähnlich wie bei den Nominalisierungen von haft-Adjektiven handelt es sich also oft um Bezeichnun-

11 Sie zeigt auch, dass kippen (im Unterschied zu abgleiten) sehr oft nicht in einer Struktur mit nominalisierten Adjektiven verwendet wird, sondern als Bewegungsverb in einem räumlichen Zusammenhang (ins Meer kippen ist die häufigste Kollokation; ins Wasser kippen ist auch sehr häufig). 
gen für Genres oder Stile, allerdings sind es viel geläufigere Bewertungen als diejenigen, die mit haft-Adjektiven vorgenommen werden, und sie sind auch meistens negativ belegt. Die Struktur 'ins + nominalisiertes Adjektiv + V' an sich wird also wohl (im Unterschied zur spezifischeren Struktur 'ins *hafte + V') nicht hauptsächlich zur Kategorisierung schwierig kategorisierbarer Veränderungsprozesse gebraucht, sondern um negative Bewertungen in Bezug auf bestimmte Tendenzen oder Veränderungen auszudrücken (die negative Konnotation mag allerdings an der Semantik dieser zwei Verben liegen).

\section{Fazit}

Bei der Struktur 'ins *hafte + V' handelt es sich um ein sprachliches Muster, das in bestimmten Textsorten, besonders Kunstrezensionen, regelmäßig vorkommt und als sprachliche Ressource angesehen werden kann, um einen noch nicht abgeschlossenen Veränderungsprozess oder eine Tendenz und die Wahrnehmung davon sprachlich zu kategorisieren und zu beschreiben. Das Muster kombiniert feste Merkmale (direktionale Präpositionalgruppe, transformatives Verb) mit vielen Besetzungsmöglichkeiten für die verschiedenen Positionen und steht damit als expressives, relativ frei variierbares Stilmittel zur Verfügung. Die Kookkurrenzdaten deuten darauf hin, dass dieses Muster auf einem allgemeineren Muster 'ins + nominalisiertes Adjektiv + V' aufbaut, das meistens allerdings weniger als anspruchsvolles Stilmittel eingesetzt wird, sondern eher relativ banale, oft negative Bewertungen in Bezug auf eine Tendenz, eine Veränderung oder die Möglichkeit einer Veränderung ausdrücken.

\section{Literatur}

Belica, Cyril (1995): Statistische Kollokationsanalyse und Clustering. Korpuslinguistische Analysemethode. Institut für Deutsche Sprache, Mannheim. www1.ids-mannheim.de/kl/ projekte/methoden/ur.html.

Chauvin, Catherine/Kauffer, Maurice (2013): La fonction expressive. Bd. 3: Écart et expressivité. Besançon: Presses universitaires de Franche-Comté.

Dalmas, Martine/Gautier, Laurent (2013): Les constructions causatives avec mouvement en allemand: d'une saisie phraséologique à une explication constructionnelle. In: Langages 189. 81-102.

Deppermann, Arnulf/Elstermann, Mechthild (2008): Lexikalische Bedeutung oder Konstruktionsbedeutungen?. In: Fischer, Kerstin/Stefanowitsch, Anatol (Hgg.): Konstruktionsgrammatik. II. Von der Konstruktion zur Grammatik. Tübingen: Stauffenburg. 103-133. 
Dobrovol'skij, Dmitrij (2011): Phraseologie und Konstruktionsgrammatik. In: Lasch, Alexander/ Ziem, Alexander (Hgg.): Konstruktionsgrammatik. III. Aktuelle Fragen und Lösungsansätze. Tübingen: Stauffenburg. 111-130.

Fabricius-Hansen, Cathrine (1975): Transformative, intransformative und kursive Verben. Tübingen: Niemeyer.

Fleischer, Wolfgang/Barz, Irmhild/Schröder, Marianne (2012): Wortbildung der deutschen Gegenwartssprache. Berlin/Boston: De Gruyter.

Gautier, Laurent/Monneret, Philippe (2010): La fonction expressive. Bd. 2. Besançon: Presses universitaires de Franche-Comté.

Goldberg, Adele (1995): Constructions: a construction grammar approach to argument structure. Chicago: The university of Chicago Press.

Legallois, Dominique/François, Jacques (2009): Définition et illustration de la notion d'expressivité en linguistique. In: Le Querler, Nicole/Neveu, Franck/Roussel, Emmanuelle (Hgg.): Relations, connexions, dépendances: hommage au professeur Claude Guimier. Rennes: Presses universitaires de Rennes. 197-221. [textes des communications présentées lors du colloque organisé à Caen, 17-18 mars 2011].

Polenz, Peter von (1988): Deutsche Satzsemantik. Grundbegriffe des Zwischen-den-ZeilenLesens. Berlin/New York: De Gruyter.

Thim-Mabrey, Christiane (2001): Grenzen der Sprache - Möglichkeiten der Sprache: Untersuchungen zur Textsorte Musikkritik. (= Regensburger Beiträge zur deutschen Sprach- und Literaturwissenschaft, Reihe B 79). Frankfurt a.M. u.a.: Lang.

Thim-Mabrey, Christiane (2007): Linguistische Aspekte der Kommunikation über Kunst. In: Hausendorf, Heiko (Hg.): Vor dem Kunstwerk: interdisziplinäre Aspekte des Sprechens und Schreibens über Kunst. München: Fink. 99-121.

Ziem, Alexander/Lasch, Alexander (2013): Konstruktionsgrammatik. Konzepte und Grundlagen gebrauchsbasierter Ansätze. (= Germanistische Arbeitshefte 44). Berlin/Boston: De Gruyter. 